\title{
Is Impulsivity a Male Trait Rather than Female Trait? Exploring the Sex Difference in Impulsivity
}

\author{
Aviv Weinstein • Pinhas Dannon
}

Published online: 15 January 2015

(C) Springer International Publishing AG 2015

\begin{abstract}
Impulsivity is raising major interest clinically because it is associated with various clinical conditions such as delinquency, antisocial behavior, suicide attempts, aggression, and criminal activity. The evolutionary perspective argued that impulsivity relates to self-regulation and it has predicted that female individuals should have evolved a greater ability to inhibit pre-potent responses. There is supportive evidence showing that female individuals have better performance on cognitive tasks measuring impulsivity such as delay in gratification and delayed discounting mainly in childhood. During adolescence, brain imaging studies using diffusion tensor imaging on white matter architecture indicated contrary to the evolutionary perspective hypothesis, that young adolescent male individuals may be less vulnerable than age-matched female individuals to risk- and reward- related maladaptive behaviors. In adults, the results are mixed presumably owing to hormonal effects on neuro-biological mechanisms of reward. Consequently, female individuals were less impulsive than male individuals only during fertile stages of the menstrual cycle. Finally, there is evidence the serotonin $(5-\mathrm{HT})$ system is more involved in the impulsivity of men than in that of women. Overall, there seem to be sex differences in impulsivity but these differences are more pronounced in childhood and they are later subject to maturational and hormonal changes during adolescence and adulthood and their effects on the brain, cognition, and behavior.
\end{abstract}

This article is part of the Topical Collection on Personality and Impulse Control Disorders

\footnotetext{
A. Weinstein

Department of Behavioral Science, University of Ariel, Science Park, Ariel 40700, Israel

P. Dannon $(\bowtie)$

Beer Yaakov Mental Health Center and Sackler School of Medicine, Tel Aviv University, Tel Aviv, Israel

e-mail: pinhasd@post.tau.ac.il
}

Keywords Impulsivity $\cdot$ Sex differences $\cdot$ Response inhibition $\cdot$ Reward

\section{Introduction}

Impulsivity generally refers to acting without forethought $[1$, 2]. It can be seen as a set of heterogeneous suboptimal behaviors along two dimensions. One component of impulsivity reflects impulsive action, which is the inability to inhibit a pre-potent response and another component is impulsive choice, which is the difficulty in delaying gratification [3]. A third component reflects impulsive decision making, such as making sub-optimal risky decisions without sufficient consideration $[4,5]$. Research on impulsivity has important implications, as impulsive behaviors greatly influence an individual's social life; its adverse outcomes can include delinquency, antisocial behaviors, addiction, suicide attempts, aggression, crimes and pathological gambling [6-10]. Theoretically, impulsivity is closely related to other personality constructs such as sensation seeking [11], failure to plan [12], lack of perseverance [13], venturesomeness [14], poor self-discipline [15], and novelty seeking [16]. For a recent review see Cross et al. [17]. This review searched articles published between 2000 and 2014 in PubMed, Ovid, and Google scholar using the key words "Impulsivity", "gender", "female impulsive behavior", and "sex differences".

\section{Neuro-biological Theories of Impulsivity}

Neuro-biological theories of personality suggest that impulsivity results from interplay between sensation and novelty seeking and harm avoidance or inhibition [18-20]. Dopamine 
is involved in reward and approach behavior, whereas serotonin mediates restraint or inhibition. Dopamine accelerates risky behavior because, when faced with danger, highsensation seekers experience stronger attraction than lowsensation seekers. Men's greater sensation seeking chiefly results from a more reactive dopaminergic system [21]. Cloninger [16] suggested three genetically mediated, independent dimensions of personality: novelty seeking, harm avoidance, and reward dependence. Cloninger used the term novelty seeking as an alternative to impulsivity, an appetitive motivation that is associated with activity in the dopaminergic reward system and is expressed as a tendency to respond to novel stimuli with excitement [22]. Novelty seeking is highly correlated with Zuckerman's sensation seeking but unlike sensation seeking, no sex difference was found for novelty seeking in a recent meta-analysis [23].

\section{The Evolutionary Perspective}

The topic of impulsivity relates to a broader area of selfregulation that has an evolutionary perspective. Selfregulation is the capacity to select actions that lead to favorable outcomes and avoid actions that lead to unfavorable outcomes. Bjorklund and Kipp [24] hypothesized that because of the different selection pressures placed on male and female individuals, female individuals should have evolved a greater ability to inhibit pre-potent responses. They have argued that overall, the evidence shows a moderate female advantage in behavioral inhibition and a strong female advantage in social inhibition [24]. Hosseini-Kamkar and Morton [25] after evaluating the behavioral, neural, and hormonal evidence for the evolutionary hypothesis have concluded that sex differences in self-regulation are more consistently reported in children prior to the onset of puberty. In adult cohorts, the results of studies examining sex differences in selfregulation were mixed.

\section{The Cognitive Assessment of Impulsivity and Sex Differences}

In the 1970s, Mischel created the delay of gratification paradigm to test children's ability to resist temptation and forgo a small immediate reward to obtain a larger delayed reward [26]. Mischel and Underwood [26] were the first to report that female preschoolers were able to wait for significantly longer periods of time to obtain the larger reward in comparison to their male counterparts. More recently, a meta-analysis was conducted on 33 experiments using the delay of gratification paradigm by Silverman [27]. The meta-analysis revealed a female advantage in the capacity to delay gratification although the effect size of the female advantage in delay of gratification is relatively small; however, this may be owing to instruments that lack precision and small sample sizes. Based on these findings, it appears as though female individuals are better suited to delay gratification and resist temptation in comparison to male individuals and that this sex difference emerges early in development.

While the results of delay of gratification measures and teacher/parent reports of self-regulation indicate that there is a female advantage in self-control (at least early in development), the results on a related measure (delay discounting) are contradictory. Delay discounting is a task used to measure impulsive choice by asking participants to select between a large reward delivered at variable delays ( 1 day, 7 days, 30 days) and a smaller immediate reward. As the length of the delayed reward increases, participants are more inclined to select the smaller immediate reward. Evolutionary theorists such as MacDonald [28] argued that male individuals are more likely to score higher on behavioral approach measures (sensation seeking, impulsivity, reward seeking, and aggression) and are thus less likely to control pre-potent approach tendencies. Dittrich and Leipold [29] reported supporting evidence that male individuals preferred a smaller immediate payment rather than a larger delayed payment thus suggesting that female individuals are better able to delay gratification in comparison to male individuals. There is contradictory evidence showing the opposite pattern of sex differences in relation to delay discounting with female individuals discounting more steeply than male individuals [30,31]. A review article investigating sex differences in impulsive choice (which refers to the tendency to prefer small immediate rewards rather than large delayed rewards) and impulsive action (a lack of behavioral inhibition) showed that many of these inconsistent findings may be due to variations in the subjects under study and the tasks used [3]. Weafer and de Wit [3] reviewed the literature and argued that sex differences appear to exist on these measures, but the direction and magnitude of the differences vary. In humans and some other species, female individuals tend to discount more steeply than male individuals on measures of delay discounting (impulsive choice), whereas sex differences in impulsive action depend on the task administered. Male individuals commit more inhibitory errors on the Go/No-Go task (failing to inhibit a pre-potent response) than female individuals, whereas female individuals commit more inhibitory errors than male individuals on the Stop Signal Task. Based on these findings and others it appears that female individuals have an advantage in terms of delay of gratification but not necessarily on impulsive action. However, the sex difference in delay of gratification and inhibitory control more generally is more evident in childhood, whereas in adults there are inconsistent results. Hosseini-Kamkar and Morton [25] argued that one potential reason for these mixed findings in adult cohorts could be because 
of the activation effects of hormones. Sex hormones may modulate the neural circuitry underlying selfregulation and result in differential patterns of behavior across different phases of the menstrual cycle. They have suggested that the mixed findings in selfregulation in adult cohorts could potentially be due to variations in levels of hormones exerting their influence on the meso-cortico-limbic dopamine reward pathway. It is therefore being suggested that individuals who have increased neural responses to rewards and/or are more sensitive to the prospect of obtaining rewards would have greater difficulty inhibiting pre-potent responses and delaying gratification.

\section{Impulsivity, Reward and Hormonal Changes}

Structural studies using autoradiography and functional studies using positron emission tomography showed that male individuals had higher $\mathrm{D}_{1}$ receptor density in the nucleus accumbens [32]; women may have lower $\mathrm{D}_{2}$ receptor occupancy compared with men [33], and this reduced $\mathrm{D}_{2}$ receptor occupancy may result in higher striatal dopamine synthesis capacity in women relative to men [34]. These sexual differences in the structure and subsequent function of the dopamine reward pathway may make men and women differentially susceptible to the reinforcing properties of reward. Munro et al. [35] revealed that men had greater dopamine release in the ventral striatum in response to amphetamines. Perhaps under baseline conditions, female individuals have greater dopamine synthesis capacity; however, when administered a rewarding substance, male individuals have greater dopamine release in the ventral striatum. Furthermore, Munro et al. [35] reported that the baseline binding potential of $\mathrm{D}_{2}$ receptors varies as a function of the menstrual cycle: women in the luteal phase had lower baseline binding potential in the putamen relative to women in the follicular phase. In the light of these findings, an investigation of hormonal influences on the dopamine reward pathway is warranted.

Recent experiments suggested that female individuals are less impulsive than male individuals only during fertile stages of the menstrual cycle. Dreher et al. [36] obtained functional magnetic resonance imaging (fMRI) scans from healthy women during the luteal and mid-follicular phases of their menstrual cycle. The fMRI paradigm included an event-related reward task; the results provided evidence of enhanced blood-oxygen-level-dependent responses associated with rewards during the phase of their menstrual cycle directly preceding ovulation. Because of the fluctuating levels of estrogen and progesterone across the menstrual cycle, female individuals may adopt differential strategies to facilitate reproduction and mate selection during different phases of their menstrual cycle. For example, being impulsive might not incur enormous risks during phases of the menstrual cycle when female individuals are not fertile any risk incurred would only apply to the female individual herself and not to a developing embryo. In accordance with this hypothesis, Pine and Fletcher [37] demonstrated that women in their luteal phase (nonfertile phase) are more impulsive than women in other phases of the menstrual cycle. However, according to parental investment theory, female individuals must be selective in securing an appropriate mate and therefore, they may exhibit less impulsivity during periods of their menstrual cycle when they have the capacity to reproduce [38-40]. These fluctuations as a result of hormonal changes during the menstrual cycle may help explain the mixed findings in delay discounting in adult cohorts that might be influenced by the phases of fertility (i.e., women discount more steeply in non-fertile phases of the menstrual cycle and discount less steeply in the fertile phase of their menstrual cycle).

Recent brain imaging studies using fMRI have also shown sex differences in impulsivity and its neural correlates. Liu et al. [41] compared male and female performance on the Go/No-Go task and found higher activation of the rostral anterior cingulate cortex (ACC) in male individuals compared with female individuals. Furthermore, male individuals showed strong negative correlations between reaction time in the impulse inhibition task and ACC and a positive correlation with ratings of excitement seeking. Interestingly, this area has been implicated in many behavioral and clinical conditions that are more prevalent in male than in female individuals such as attention-deficit hyperactivity disorder, violence, and aggression.

The ability to resist reward-related impulses was investigated by Diekhof et al. [42] who assessed with fMRI which neural processes enable men and women to successfully control their desire for immediate reward when this is required by a higher-order goal (i.e., during a "desire-reason dilemma") [43]. Although men and women showed similarities in the general response of reward areas such as the nucleus accumbens and the ventral tegmental area to predictors of immediate reward, they differed in additional brain mechanisms that enabled self-controlled decisions against the preference for immediate reward. First, men exhibited a stronger reduction in activation in the ventral pallidum, putamen, temporal pole, and pregenual ACC during the "desirereason dilemma". Second, connectivity analyses revealed a significant change in the direction of the connectivity between the prefrontal cortex and nucleus accumbens during decisions counteracting the reward-related impulse when comparing men and women. Altogether, these studies support the view of sex differences in the recruitment of sex-specific neural resources during the successful deployment of self-control, inhibition, and delayed gratification and merit further investigation. 


\section{Impulsivity, White Matter Architecture and Sex Differences During Adolescence}

During adolescence, rapid maturational brain changes occur at a time that is associated with risk-taking behaviors and cognitive development. Studies on sex differences, impulsive behavior, and white matter architecture in adolescents using diffusion tensor imaging were reviewed by Bava et al. [44]. Silveri et al. [45] reported sex differences in fractional anisotropy (FA), a measure of white matter coherence were related to response inhibition measured by the Stroop task. A following study revealed that greater white matter anisotropy in several areas in male individuals was associated with lower impulsivity measured by a delay-discounting task [46]. Finally, maturation of medial frontal cortices in male individuals was implicated in the maturation of rational decision making and avoidance of risky choices [47]. Higher FA measures in male individuals in frontal, fronto-parietal, and fronto-temporal white matter pathways were found and these sex differences in association fibers may be associated with differential vulnerabilities to maladaptive behaviors, considering their link to risk-taking tendencies [44]. Given that risky and sensationseeking behavior peaks relative to puberty [48, 49], it has been argued that young adolescent male individuals may be less vulnerable than age-matched female individuals to risk- and reward-related maladaptive behaviors [44]. Sex differences in brain structure and impulsivity during adolescence may have implications for risky sexual behavior among adolescent female individuals [50]. Research and treatment should therefore consider gender differences when investigating the role of impulsivity in adolescent sexual risk-taking behavior.

\section{Impulsivity, Serotonin (5-HT) and Sex Differences}

Although dopamine and reward have been widely investigated in the context of impulsivity and sex differences, the brain's serotonin (5-HT) system has a crucial role in impulsivity. 5HT plays an important role in the modulation of different functions and behaviors including appetite, sleep, memory and learning, mood and sexuality [51, 52]. However, one of the most important roles of 5-HT in the central nervous system is that it might serve as a "modulator" of impulsivity and aggression [53-57]. In a preliminary study, Marazziti et al. [58] explored the relationships between impulsivity, sex and a peripheral serotonergic marker, the platelet serotonin transporter (SERT), in a group of 32 healthy subjects. They found that women had a higher Barratt Impulsivity Scale (BIS-11) total score than men, and also higher scores of two factors of the same scale: the motor impulsivity and the cognitive complexity. Second, the density of the SERT transporter was significantly and positively related to the cognitive complexity factor, but only in men. Furthermore, men showed a significant and negative correlation with 5-HT binding, and the motor impulsivity factor. These findings suggest that women are generally more impulsive than men, but that the 5-HT system is more involved in the impulsivity of men than in that of women.

\section{Summary}

There are many indicators that from the evolutionary and neuro-biological perspectives men are more impulsive than women who should have evolved a greater ability to inhibit pre-potent responses. There is supporting evidence showing that women perform better on cognitive tasks measuring impulsivity such as delay in gratification and delayed discounting mainly in childhood. During adolescence, brain imaging studies indicated that contrary to the evolutionary perspective hypothesis, young adolescent male individuals may be less vulnerable than age-matched females to riskand reward- related maladaptive behaviors. During adulthood, there are mixed results on sex differences in impulsivity that could be owing to hormonal changes and their effects on the dopamine reward system. There is evidence that the 5-HT system is more involved in the impulsivity of men than in that of women. The sex difference in impulsivity is therefore subject to maturational, neuro-pharmacological and hormonal changes during adolescence and adulthood. The emerging evidence for the genetic role in impulsivity $[2,59,60]$ could also perhaps explain sex differences in impulsivity in the future.

\section{Compliance with Ethics Guidelines}

Conflict of Interest Aviv Weinstein and Pinhas Dannon have no conflicts of interest.

Human and Animal Rights and Informed Consent This article does not contain any studies with human or animal subjects performed by any of the authors.

\section{References}

1. Cardinal RN. Neural systems implicated in delayed and probabilistic reinforcement. Neural Netw. 2006;19(8):1277-301.

2. Bevilacqua L, Goldman D. Genetics of impulsive behaviour. Philos Trans R Soc Lond B Biol Sci. 2013;368(1615):20120380.

3. Weafer J, de Wit H. Sex differences in impulsive action and impulsive choice. Addict Behav. 2014;39(11):1573-9.

4. Bechara A, Damasio AR, Damasio H, Anderson SW. Insensitivity to frontal cortex following damage to human prefrontal cortex. Cognition. 1994;50:7-15.

5. Lejuez CW, Read JP, Kahler CW, Richards JB, Ramsey SE, Stuart GL, et al. Evaluation of a behavioral measure of risk-taking: the balloon analogue risk task (BART). J Exp Psychol. 2002;8:75-84. 
6. Flory JD, Harvey PD, Mitropoulou V, New AS, Silverman JM, Siever LJ, et al. Dispositional impulsivity in normal and abnormal samples. J Psychiatry Res. 2006;40(5):438-47.

7. John OP, Caspi A, Robins RW, Moffitt TE, Stouthamer-Loeber M. The 'Little Five': exploring the nomological network of the fivefactor model of personality in adolescent boys. Child Dev. 1994;65: 160-78.

8. Luengo MA, Carrillo-de-la-Peña MT, Otero JM, Romero E. A shortterm longitudinal study of impulsivity and antisocial behavior. J Pers Soc Psychol. 1994;66(3):542-8.

9. Tremblay RE, Pihl RO, Vitaro F, Dobkin PL. Predicting early onset of male antisocial behavior from preschool behavior. Arch Gen Psychiatry. 1994;51:732-39.

10. Wong G, Zane N, Saw A, Chan AK. Examining gender differences for gambling engagement and gambling problems among emerging adults. J Gambl Stud. 2013;29(2):171-89.

11. Zuckerman M. Sensation seeking: beyond the optimal level of arousal. Hillsdale: Erlbaum; 1979.

12. Patton JH, Stanford MS, Barratt ES. Factor structure of the Barratt Impulsiveness Scale. J Clin Psychol. 1995;51:768-74.

13. Whiteside SP, Lynam DR. The five factor model and impulsivity: using a structural model of personality to understand impulsivity. Personal Individ Differ. 2001;30:669-89.

14. Eysenck HJ, Eysenck MW. Personality and individual differences: a natural science approach. New York: Plenum Press; 1985.

15. Costa Jr PT, McCrae RR. Revised NEO Personality Inventory (NEOPI-R) and NEO Five Factor Inventory (NEO-FFI) professional manual. Odessa: Psychological Assessment Resources; 1992.

16. Cloninger $\mathrm{CR}$. A systematic method for clinical description and classification of personality variants: a proposal. Arch Gen Psychiatry. 1987;44:573-88.

17. Cross CP, Copping LT, Campbell A. Sex differences in impulsivity: a meta-analysis. Psychol Bull. 2011;137(1):97-130.

18. Zuckerman M. Personality in the third dimension: a psychobiological approach. Personal Individ Differ. 1989;10:391-418.

19. Zuckerman M. Behavioral expressions and biosocial bases of sensation seeking. New York: Cambridge University Press; 1994.

20. Zuckerman M. Sensation seeking and risky behavior. Washington, DC: American Psychological Association; 2007.

21. Zuckerman M, Kuhlman DM. Personality and risk-taking: common biosocial factors. J Pers. 2000;68:999-1029.

22. Cloninger CR. A unified biosocial theory of personality and its role in the development of anxiety states. Psychiatr Dev. 1986;4:167-226.

23. Miettunen J, Veijola J, Lauronen E, Kantojärvi L, Joukamaa M. Sex differences in Cloninger's temperament dimensions: a meta-analysis. Compr Psychiatry. 2007;48:161-9.

24. Bjorklund DF, Kipp K. Parental investment theory and gender differences in the evolution of inhibition mechanisms. Psychol Bull. 1996;120:163-88

25. Hosseini-Kamkar N, Morton J. Sex differences in self-regulation: an evolutionary perspective. Front Neurosci. 2014;8(233):1-8.

26. Mischel W, Underwood B. Instrumental ideation in delay of gratification. Child Dev. 1974;45:1083-8.

27. Silverman I. Gender differences in delay of gratification: a metaanalysis. Sex Roles. 2003;49:451-63.

28. MacDonald KB. Effortful control, explicit processing and the regulation of human evolved predispositions. Psychol Rev. 2008;115: 1012-31.

29. Dittrich M, Leipold K. Gender differences in time preferences. Econ Lett. 2014;122:413-5.

30. Reynolds B, Ortengren A, Richards J, Dewit H. Dimensions of impulsive behavior: personality and behavioral measures. Personal Individ Differ. 2006;40:305-15.

31. Beck RC, Triplett MF. Test-retest reliability of a group-administered paper-pencil measure of delay discounting. Exp Clin Psychopharmacol. 2009;17:345-55.
32. Andersen SL, Rutstein M, Benzo JM, Hostetter JC, Teicher MH. Sex differences in dopamine receptor overproduction and elimination. Neuroreport. 1997;8:1495-7.

33. Pohjalainen T, Rinne J, Nagren K, Syvalahti E, Hietala J. Sex differences in the striatal dopamine D2 receptor binding characteristics in vivo. Am J Psychiatr. 1998;155:768-77.

34. Laakso A, Vilkman H, Bergman J, Haaparanta M, Solin O, Syvalahti E, et al. Sex differences in striatal presynaptic dopamine synthesis capacity in healthy subjects. Biol Psychiatry. 2002;52:759-63.

35. Munro CA, Ye W, Wand GS, Alexander M, Kumar A, Kuwabara H, et al. Sex differences in striatal dopamine release in healthy adults. Biol Psychiatry. 2006;59:966-74.

36. Dreher J, Schmidt PJ, Kohn P, Furman D, Rubinow D, Berman KF. Menstrual cycle phase modulates reward-related neural function in women. Proc Natl Acad Sci U S A. 2007;104:2465-70.

37. Pine KJ, Fletcher B. Women's spending behaviour is menstrual-cycle sensitive. Personal Individ Differ. 2011;50:74-8.

38. Carroll ME, Kohl EA, Johnson KM, Lanasa RM. Increased impulsive choice for saccharin during PCP withdrawal in female monkeys: influence of menstrual cycle phase. Psychopharmacology. 2013;227: 413-24.

39. Khaighobadi F, Stevens J. Does fertility status influence impulsivity and risk taking in human females? Adaptive influences on intertemporal choice and risky decision making. Evol Psychol. 2013;11:700-17.

40. Smith C, Sierra Y, Oppler S, Boettiger C. Ovarian cycle effects on immediate reward selection bias in humans: a role for estradiol. J Neurosci. 2014;34:5468-76.

41. Liu J, Zubieta JK, Heitzeg M. Sex differences in anterior cingulate cortex activation during impulse inhibition and behavioral correlates. Psychiatry Res. 2012;201(1):54-62.

42. Diekhof EK, Keil M, Obst KU, Henseler I, Dechent P, Falkai P, et al. A functional neuroimaging study assessing gender differences in the neural mechanisms underlying the ability to resist impulsive desires. Brain Res. 2012;1473:63-77.

43. Diekhof EK, Gruber O. When desire collides with reason: functional interactions between anteroventral prefrontal cortex and nucleus accumbens underlie the human ability to resist impulsive desires. J Neurosci. 2010;30(4):1488-93.

44. Bava S, Boucquey V, Goldenberg D, Thayer RE, Ward M, Jacobus J, et al. Sex differences in adolescent white matter architecture. Brain Res. 2011;1375:41-8.

45. Silveri MM, Rohan ML, Pimentel PJ, Gruber SA, Rosso IM, Yurgelun-Todd DA. Sex differences in the relationship between white matter microstructure and impulsivity in adolescents. Magn Reson Imaging. 2006;24:833-41.

46. Olson EA, Collins PF, Hooper CJ, Muetzel R, Lim KO, Luciana M. White matter integrity predicts delay discounting behavior in 9- to 23-year-olds: a diffusion tensor imaging study. J Cogn Neurosci. 2009;21:1406-21.

47. Crone EA, Bullens L, van der Plas EA, Kijkuit EJ, Zelazo PD. Developmental changes and individual differences in risk and perspective taking in adolescence. Dev Psychopathol. 2008;20: 1213-29.

48. Steinberg L. Risk taking in adolescence: what changes, and why? Ann N Y Acad Sci. 2004;1021:51-8.

49. Steinberg L. A social neuroscience perspective on adolescent risktaking. Dev Rev. 2008;28:78-106.

50. Dir AL, Coskunpinar A, Cyders MA. A meta-analytic review of the relationship between adolescent risky sexual behavior and impulsivity across gender, age, and race. Clin Psychol Rev. 2014;34(7):551-62.

51. Evenden JL. Varieties of impulsivity. Psychopharmacology. 1999;146:348-61.

52. Olivier B. Serotonin and aggression. Ann N Y Acad Sci. 2004;1036: 382-92. 
53. Soubri P. Reconciling the role of central serotonin neurons in human and animal behavior. Behav Brain Sci. 1986;9: 319-64.

54. Highley JD, Linnoila M. Low central nervous system serotonergic activity is trait like and correlates with impulsive behavior. Ann N Y Acad Sci. 1997;836:39-56.

55. Kalenscher T, Ohmann T, Gunturkun O. The neuroscience of impulsive and self-controlled decisions. Int J Psychophysiol. 2006;62(2): 203-11.

56. Serretti A, Mandelli L, Lorenzi C, Landoni S, Calati R, Insacco C, et al. Temperament and character in mood disorders: influence of
DRD4, SERTPR, TPH and MAO-A polymorphisms. Neuropsychobiology. 2006;53:9-16.

57. Pattij T, Vanderschuren LJ. The neuropharmacology of impulsive behaviour. Trends Pharmacol Sci. 2008;29(4):192-9.

58. Marazziti D, Baroni S, Masala I, Golia F, Consoli G, Massimetti G, et al. Impulsivity, gender, and the platelet serotonin transporter in healthy subjects. Neuropsychiatr Dis Treat. 2010;6:9-15.

59. Congdon E, Canli T. Neurogenetic approach to impulsivity. J Pers. 2008;76(6):1447-84.

60. Wong NML, Lee TMC. Genetic and neural relationships underpinning impulsivity. Int J Psychol Res. 2013;6:80-93. 